\title{
Phase transition in strongly stretched polymer gels
}

\author{
A. Suzuki, K. Sanda, and Y. Omori \\ Department of Materials Science, Yokohama National University, 79-5 Tokiwadai, Hodogaya-ku, \\ Yokohama 240, Japan
}

(Received 31 March 1997; accepted 25 June 1997

\begin{abstract}
We will present the volume phase transition of cylindrical pAlyisopropylacrylamide (NIPA) gels under large distortion along the uniaxial direction. The neutral and slightly ionized NIPA gels were synthesized in submillimeter diameter. The gel was stretched along the uniaxial direction with the longitudinal deformation ratio $\alpha_{\|}$(the ratio of the stretched length to the equilibrium length in the swollen state at $30^{\circ} \mathrm{C}$ ). By keeping the longitudinal length constant, we have simultaneously measured the equilibrium diameter and the force as functions of temperature. The swelling curves of the neutral NIPA gels including the volume phase transition temperature were obtained for several deformations in the range between $\alpha_{\|}=1$ and $\alpha_{\|}=6$. With increasing $\alpha_{\|}$, the transition temperature increased up to $1{ }^{\circ} \mathrm{C}$ in the small deformation below $\alpha_{\|} \sim 3.5$, and it saturated and slightly decreased in the large $\alpha_{\|}$above $\alpha_{\|} \sim 4$. At the transition temperature on heating, the force to keep the length constant increased discontinuously in the smaller region below $\alpha_{\|} \sim 3.5$. The magnitude of this steplike change in the force began to decrease in the vicinity of $\alpha_{\|} \sim 3.5$, and hereafter exhibited negative change above $\alpha_{\|} \sim 4$. In the case of the slightly ionized NIPA gels, these effects came into play at an earlier stage, that is, at smaller $\alpha_{\|}$, because of the prestretching due to the ionic pressure. The present observations, especially for the strong deformation above $\alpha_{\|} \sim 4$, were discussed by the equation of states of strongly stretched hydrophobic gels on the basis of the extended Flory-type free energy taken into account the non-Gaussian effect. (C) 1997 American Institute of Physics. [S0021-9606(97)50737-9]
\end{abstract}

\section{INTRODUCTION}

Polymer gels are made of cross-linked networks of polymers and liquids, and have solidlike and liquidlike behavior due to the elastic as well as osmotic nature. The interaction between polymer and solvent, which determines the structural properties and the unique functions of gels, has been studied extensively in a number of systems exhibiting the volume phase transition. ${ }^{1,2}$ Among the physical and chemical properties of such systems, mechanical response of polymer gels has been usually examined in the linear regime, where cross-link points are uniformly deformed. ${ }^{3,4}$ On the other hand, in the strong deformation, many chains should be stretched to something approaching the full length of the chain, then one should take into account such effects as well as the deviations of the cross-link points from uniform deformation. It is important to investigate experimentally the limit of strong deformation, since the stress is believed to be theoretically sensitive to the nature of the individual chains which has the non-Gaussian character. ${ }^{5,6}$ Recently, we established experimentally the static properties of mechanically constrained poly $N$-isopropylacrylamide (NIPA) gels through the phase transition behavior. ${ }^{7}$ We have measured the diameter and the force of cylindrical NIPA gels of submillimeter diameter ${ }^{8,9}$ under mechanical distortion along the uniaxial direction in the smaller elongation ratio. Both the transition temperature and force increased with increasing the fixed length along the uniaxial direction. The elastic response in the smaller deformation were quite successfully described by the phenomenological model on the basis of the extended Flory-type free energy. ${ }^{3}$ One can say that the experimental observation and the consistence with the theory should result from the fact that the deformation was small enough not to evidently disturb the assumption of the Gaussian behavior of the polymer networks. There exist several theoretical explanations for the non-Gaussian behavior ${ }^{10-13}$ on the large deformations of polymer networks, while a few experimental studies related to the phase transition of polymer gels have been reported only for small distortion of gels, ${ }^{3,4,7,9}$ and no experimental work for much larger distortion seems to be available at present. The understanding of the mechanism of the response to strong deformation is still not complete.

In this paper, we report further details of the static properties of the neutral NIPA gels of submillimeter diameter mechanically constrained under large elongation ratio along the uniaxial direction. The NIPA gels are well known to exhibit the volume phase transition in response to temperature, ${ }^{14,15}$ and its mechanism has been attributed to the hydrophobic interaction. We will present the changes in the diameter and the force of the neutral NIPA gel under network traction versus temperature during the phase transition between the swollen and collapsed states, as well as those of slightly ionized NIPA gels to investigate the effect of ionization. The effect of large stretch on the swelling behavior and the force change during the volume phase transition for the neutral and slightly ionized hydrophobic gels are discussed in qualitative terms with references to the non-Gaussian theory. ${ }^{16,17}$ 


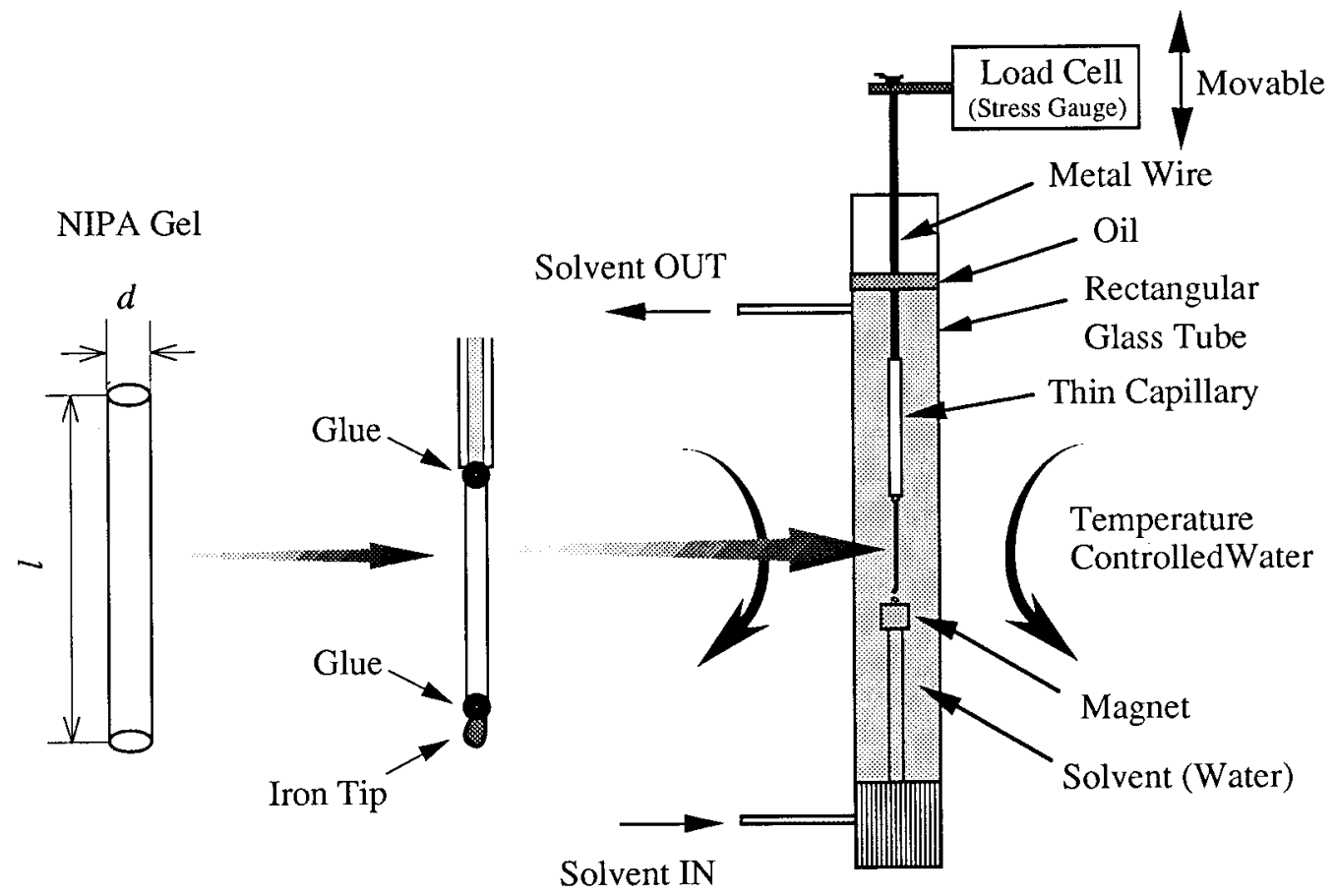

FIG. 1. The schematics of sample setup and the cell for measuring the diameter and force, and their responses to the temperature change of the cylindrical NIPA gels with submillimeter diameter. The cell was designed to circulate the solvent water in the inner rectangular capillary, out of which the temperature controlled water can be circulated.

\section{EXPERIMENTS}

Gels were prepared by a free radical copolymerization in water under nitrogen atmosphere at $0{ }^{\circ} \mathrm{C}$. The NIPA monomer was purified by recrystallization from the mixture of petroleum ether and toluene. The reagents and their amounts for the neutral NIPA gels were similar to the standard one; ${ }^{14}$ $7.8 \mathrm{~g}$ of purified NIPA (main constituent, Kodak), $0.133 \mathrm{~g}$ of $N, N^{\prime}$-methylene-bis-acrylamide (cross linker, Wako), and $240 \mu \mathrm{l}$ of $N, N, N^{\prime}, N^{\prime}$-tetramethylethylenediamine (acceralator, Wako) were dissolved in $100 \mathrm{~g}$ of water. After the solution was fully saturated with nitrogen, ammonium persulfate (Wako, $40 \mathrm{mg}$ ) as an initiator was added to the solution. Thin capillaries with an inner diameter of $141.5 \mu \mathrm{m}$ were inserted into the solution, ${ }^{9}$ which was used to make a uniform cylindrical shape. The pre-gel solution was brought into the capillaries with capillary action. The jellying solution was stored at the ice temperature overnight, in order for the gelation process to assume completion. After gelation was completed, the cylindrical gels were put out of the capillaries, and then immersed in a large amount of deionized, distilled water to wash away residual chemicals and unreacted monomers from the polymer networks, hereafter gels were placed in clean air to be dried. The neutral NIPA gels without constraint used in the present experiment exhibit the phase transition at 33.45 $( \pm 0.05){ }^{\circ} \mathrm{C}$ on increasing temperature. Slightly ionized NIPA gels were prepared by exactly the same method as the neutral NIPA gels except the amount of the main constituent. In the pre-gel solution a small amount $(1.16 \%)$ of total NIPA monomers were replaced by sodium acrylate (ionizable monomer, Nacalai Tesque).
The experimental setup was similar to that used in Ref. 7; one end of the dried gel was fixed to the capillary, while on the other end a small iron tip was attached, both by using silicone glues. The gel was inserted into the rectangular capillary with an inner size of $5 \times 5 \mathrm{~mm}^{2}$ filled with water, thus the iron tip can be fixed to the magnet (Fig. 1). In the present setup, the inner rectangular capillary was modified in order to circulate the solvent water, since the volume of the ionized NIPA gels is very sensitive to the ionic concentration, therefore it is necessary to keep the $p \mathrm{H}$ of water constant. The rectangular glass tube was encapsulated in a transparent cell. Temperature controlled water with an accuracy of $\pm 0.05{ }^{\circ} \mathrm{C}$ was circulated in the cell. After the gel reached equilibrium at each temperature, the diameter and the force were measured by an optical microscope and a load cell with a stress gauge, respectively. A 20 min interval was enough for the gel to reach thermally swelling equilibrium except near the transition point. ${ }^{7-9}$ The swelling ratio, defined as $V / V_{0}$, was calculated as $\left(d / d_{0}\right)^{2}\left(l / l_{0}\right)$ where $V, d$, and $l$ are the swollen volume, diameter, and length of the gel, respectively, and $V_{0}, d_{0}$, and $l_{0}$ are the initial respective values as prepared. All of the gels were stretched in the swollen state at $30^{\circ} \mathrm{C}$. We define the longitudinal deformation $\alpha_{\|}$as the ratio of the stretched length to the equilibrium length without stress at $30{ }^{\circ} \mathrm{C}$, and the corresponding transverse deformation is $\alpha_{\perp}$. It should be noted that the diameter of the neutral NIPA gels at $30^{\circ} \mathrm{C}$ without mechanical constraint is almost the same as the inner diameter of the capillary used in the gel synthesis, $d_{0}\left(d / d_{0}=1\right)$, therefore we used $\alpha_{\|}=l / l_{0}$ and $\alpha_{\perp}=d / d_{0}$ for the neutral NIPA gels. In the case of the ionized NIPA gels, 
the diameter at $30^{\circ} \mathrm{C}$ is larger than $d_{0}$, as $d / d_{0}=1.2$, because of the prestretching due to the ionic pressure, therefore we consider the elongation as $\alpha_{\|}=l / 1.2 l_{0}$ and $\alpha_{\perp}$ $=d / 1.2 d_{0}$. Note that for a technical reason it was difficult to use the same gel for all deformations, the gel was changed after a few sets of deformations. Nevertheless, one can believe that it will not disturb the analysis, since all the gels used in this experiment were synthesized from the same solution at the same time.

\section{RESULTS AND DISCUSSION}

\section{A. Diameter and force of stretched neutral NIPA gels versus temperature}

The equilibrium diameter and the swelling ratio of the neutral NIPA gels are shown in Fig. 2, where the temperature was increased under a constrained state for each value of the extension ratio, $\alpha_{\|}$. In both swollen and collapsed states the diameter decreases with $\alpha_{\|}$[Fig. 2(a)]. On the other hand, the swelling ratio $V / V_{0}$ of the swollen phase increases with $\alpha_{\|}$, and that of the collapsed phase is almost constant or slightly increases [Fig. 2(b)]. The transition temperature $T_{C}$ increases with $\alpha_{\|}$, and the magnitude of the steplike change of $\Delta V / V_{0}$ at the transition point becomes larger. It should be noted that the larger the deformation above $\alpha_{\|} \sim 4$, the smaller the decrement of the diameter at $30^{\circ} \mathrm{C}$, which corresponds to the fact that the increment of $T_{C}$ becomes smaller at larger $\alpha_{\|}$. The force, $F$, to keep the gel in a given $\alpha_{\|}$was simultaneously measured versus temperature. The equilibrium $F$, and the stress $\sigma$ of the neutral NIPA gels are shown in Fig. 3. The larger $\alpha_{\|}$produces the larger $F$ both in the swollen and collapsed phases, while $F$ is not sensitive to the temperature change except the transition point [Fig. 3(a)]. For the smaller $\alpha_{\|}, F$ increases discontinuously at the transition point. The magnitude of the steplike change, however, decreases as $\alpha_{\|}$is approaching to $\alpha_{\|} \sim 3.5$, and finally the change becomes continuous around $\alpha_{\|} \sim 3.5$. On the other hand, with further increasing $\alpha_{\|}$in the larger deformation above $\alpha_{\|} \sim 4$, it began to decrease at the transition point, followed by a large discontinuous decrement. In Fig. 3(b), the stress $\sigma$ (the force per unit area of the cross section) is plotted against temperature, which was calculated from the force [Fig. 3(a)] and the diameter [Fig. 2(a)] of each temperature. With increasing the temperature, $\sigma$ increases slightly in the swollen state and discontinuously at the transition point, followed by a slight increase in the collapsed phase. The magnitude of the steplike change in the stress $\Delta \sigma$ at the transition temperature becomes larger with $\alpha_{\|}$.

\section{B. Effect of the ionization of the polymer networks}

The equilibrium diameter and the force of the slightly ionized NIPA gels have been obtained as a function of temperature. The diameter decreases with $\alpha_{\|}$at a fixed temperature both in the swollen and collapsed phases, which is qualitatively the same as the result for the neutral NIPA gels [as is shown in Fig. 2(a)]. This evidence indicates that the Poisson ratio is always positive for both gels. It should be noted that the theoretical prediction of the negative Poisson ratio ${ }^{13}$
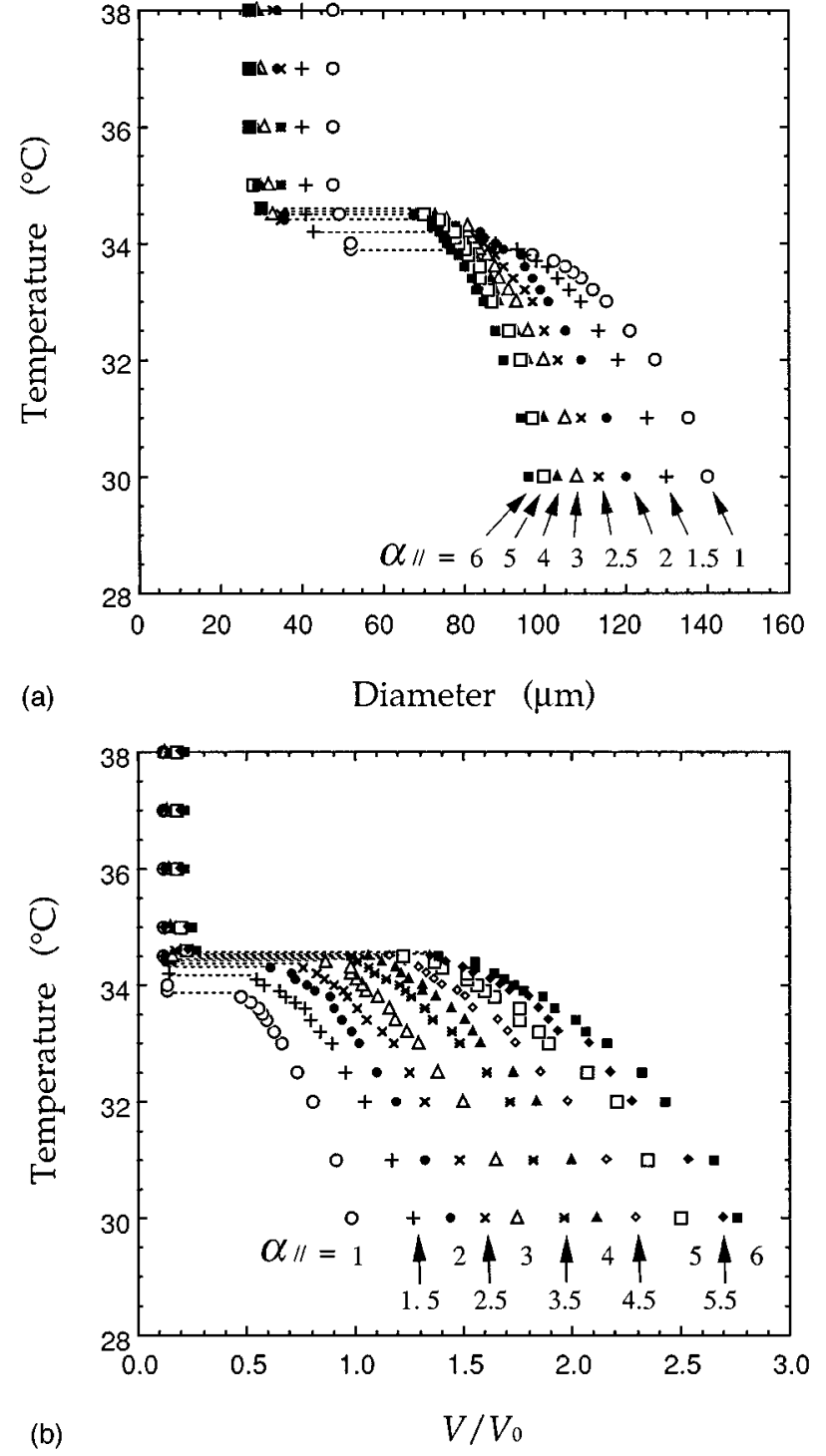

FIG. 2. The equilibrium diameter versus temperature on heating of the neutral NIPA gels in each fixed $\alpha_{\|}$(a) and the swelling ratio $V / V_{0}$ that equals $\alpha_{\|}\left(d / d_{0}\right)^{2}$ (b). The broken lines indicate the discontinuous phase transition. The error bars are smaller than the symbols.

could not be observed in the present experimental conditions. This is partly because not only the neutral but also the slightly ionized NIPA gels are not in the good solvent conditions in the present temperature range above $30^{\circ} \mathrm{C}$. One can test the theory ${ }^{13}$ for the highly ionized NIPA gels.

In order to discuss the effect of the ionization on the swelling behavior, we present $T_{C}$, and $\Delta V / V_{0}$ (the relative magnitude of the discontinuity in the swelling curve at the transition temperature) as well as $\Delta \sigma / \sigma_{0}$ (the stress jump at the transition temperature), calculated from Figs. 2 and 3. In Fig. 4(a), $T_{C}$ on heating is plotted against $\alpha_{\|}$. As is shown in this figure, $T_{C}$ of the neutral NIPA gels increases linearly with $\alpha_{\|}$in the smaller region below $\alpha_{\|} \sim 3.5$; the increment $\Delta T_{C}=0.4\left(\alpha_{\|}-1\right){ }^{\circ} \mathrm{C}$. At larger $\alpha_{\|}$it saturated to approaching a constant and exhibits a slight decrease. In the case of the slightly ionized NIPA gels, these effects came into play at an earlier stage, that is smaller $\alpha_{\|}$, because of the pre- 


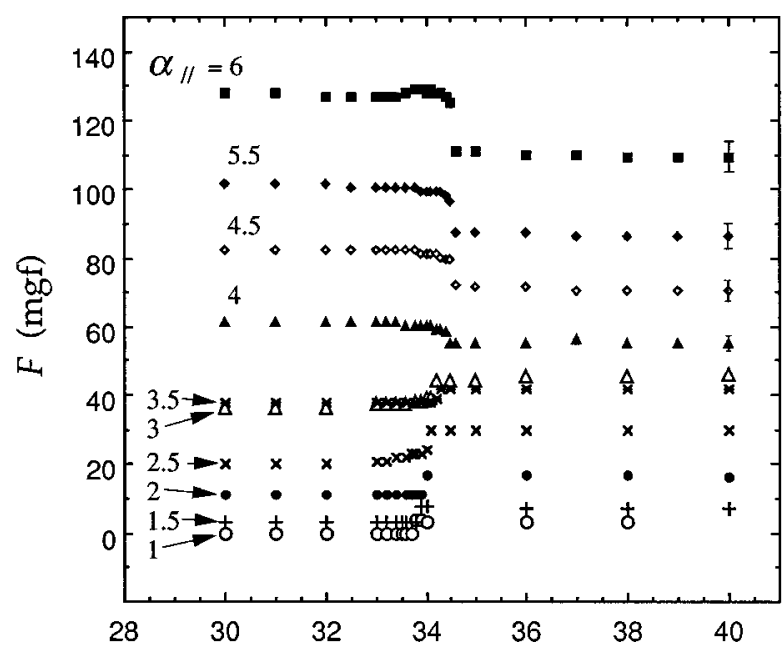

(a)

Temperature $\left({ }^{\circ} \mathrm{C}\right)$

$\left(\times 10^{6}\right)$

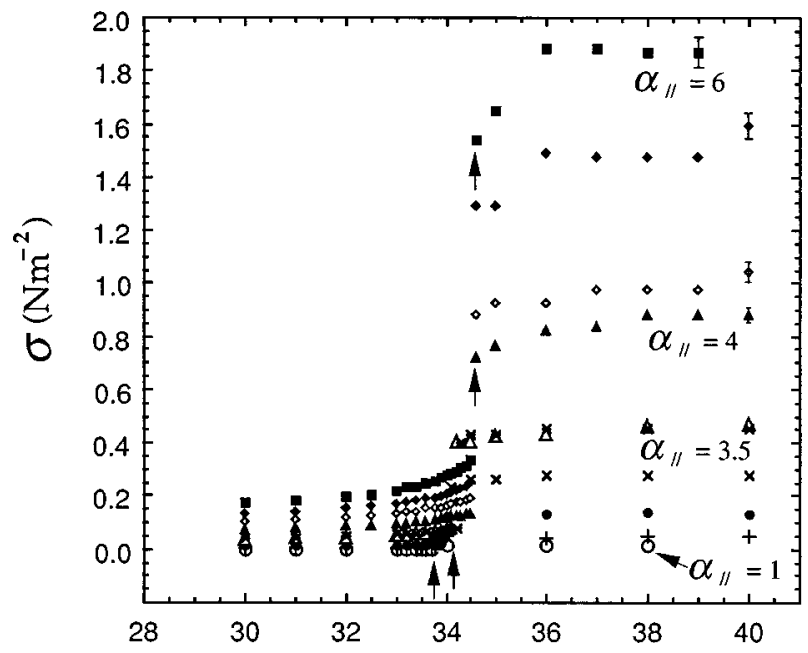

(b)

Temperature $\left({ }^{\circ} \mathrm{C}\right)$

FIG. 3. The force $F$ to keep the NIPA gel in each fixed $\alpha_{\|}$versus temperature on heating (a) and the stress $\sigma$ that equals $F / \pi(d / 2)^{2}$ (b). The arrows denote the transition point. Symbols in (b) are the same as those in (a). One error bar on each symbol denotes the typical deviation of the repeated measurements for the respective $\alpha_{\|}$. The error bars not shown are smaller than the symbols.

stretching due to the ionic pressure; $T_{C}$ increases linearly with $\alpha_{\|}, \Delta T_{C}=0.5\left(\alpha_{\|}-1\right){ }^{\circ} \mathrm{C}$, and saturated at $\alpha_{\|} \sim 2.5$. This is attributed to the aforementioned fact that the diameter of the slightly ionized NIPA gel at $30^{\circ} \mathrm{C}$ increases about 1.2 times larger than that of the neutral NIPA gels, therefore $T_{C}$ of the former increases more steeply than that of the latter. Similar features can be observed in the changes of $\Delta V / V_{0}$ and $\Delta \sigma / \sigma_{0}$ [Fig. 4(b)]: $\Delta V / V_{0}$ increases linearly with $\alpha_{\|}$and saturated at larger $\alpha_{\|}$, while $\Delta \sigma / \sigma_{0}$ gradually increased at smaller $\alpha_{\|}$and rapidly at larger $\alpha_{\|}$. All these consistent observations of the parameter changes indicate that there is a crossover deformation ratio, $\alpha_{\|}^{*}\left(3.5<\alpha_{\|}^{*}<4\right.$ for the pure NIPA gel), and some complement effects should have an

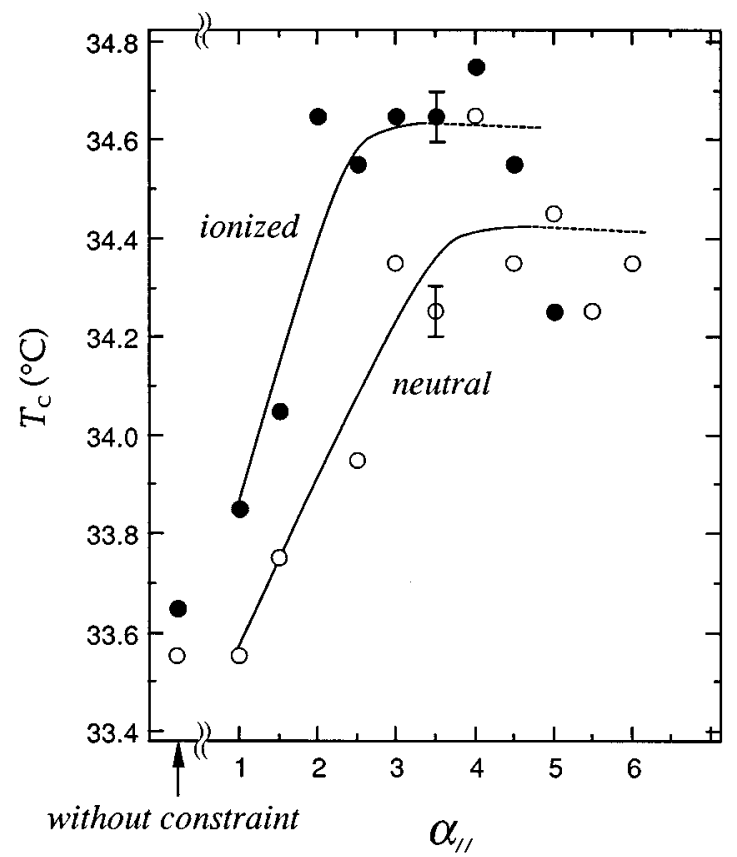

(a)

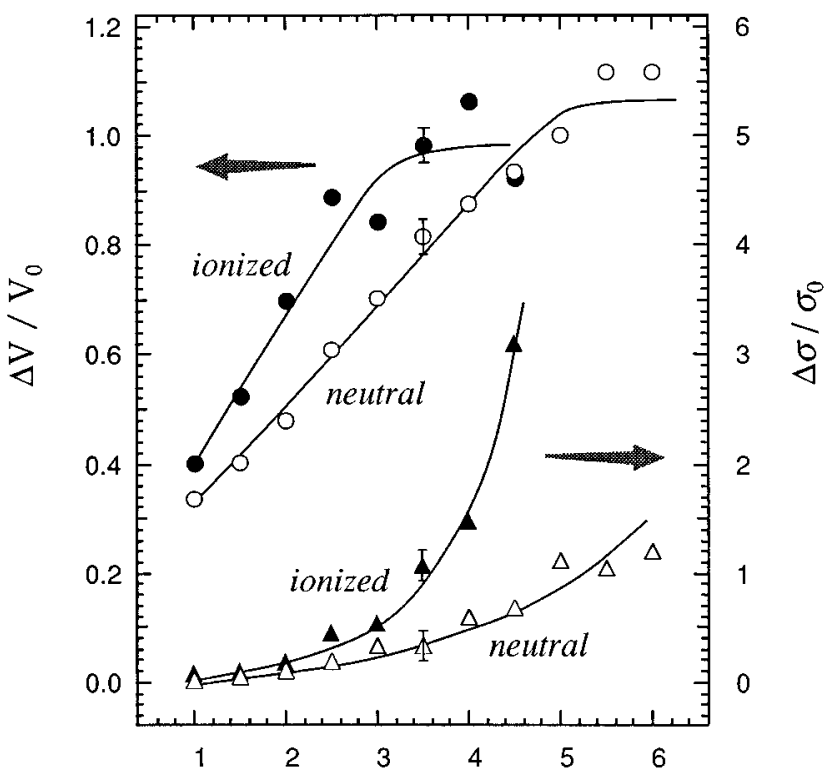

(b)

$$
\alpha_{1 /}
$$

FIG. 4. The transition temperature $T_{C}$ of the neutral NIPA gels and ionized NIPA gels on heating as functions of $\alpha_{\|}$(a) and the transition width of $V / V_{0}$ and $\sigma / \sigma_{0}(\mathrm{~b})$, where $V_{0}$ is the initial volume at gelation, and $\sigma_{0}=1$ $\times 10^{6}\left(\mathrm{~N} \mathrm{~m}^{-2}\right)$. Lines added are to guide the eye. One error bar on each symbol denotes the typical deviation of the repeated measurements for the respective curve.

important role at larger than $\alpha_{\|}^{*}$, which will be discussed later.

The ionized NIPA gel used here is incorporated with only a small amount of ionizable monomers (sodium acrylate). We believe that the perturbation due to the incorporation of the monomers does not affect the network structures of the pure NIPA gel, which results in the observation of the prestretching effect in the present study. In the case of the 
high concentration of the ionizable monomers, it is important to take into account the effect of the incorporation with the ionic monomers on the network structure as well as the amount of disorders.

\section{Equation of states of strongly stretched cylindrical gels}

There appeared two interesting features in the swelling curves of the strongly stretched NIPA gels. One is that $T_{C}$ increases linearly with $\alpha_{\|}$in the smaller region below $\alpha_{\|}^{*}$. It saturated to approaching a constant when the elongation was increased above $\alpha_{\|}^{*}$, and the other is that the force $F$ increased at the transition point in the smaller $\alpha_{\|}$region, while it decreased above $\alpha_{\|}^{*}$. It has been pointed out that the elastic behavior of polymer networks deviates from Gaussian behavior under a uniaxial deformation. ${ }^{16-21}$ Even though the elastic properties have been well described by the mean-field theory based on the Gaussian model in the smaller $\alpha_{\|}$, and we have demonstrated the validity in the previous paper. In the case of the strongly stretched neutral NIPA gel, we extended the calculation at the larger $\alpha_{\|}$region, and found that $T_{C}$ increases monotonically even in the larger $\alpha_{\|}$, and $F$ increased at the phase transition point under the larger $\alpha_{\|}$. Therefore we cannot use the model to explain the present observation. Now we will examine the above features and attempt here the qualitative explanations within the meanfield theory. The free energy of the system consists of two independent contributions of osmotic term (mixing free energy), $\Delta G_{\text {mix }}$ and the elastic term (elastic energy), $\Delta G_{\mathrm{el}}$ based on the Flory-Huggins theory, $\Delta G=\Delta G_{\text {mix }}+\Delta G_{\mathrm{el}}$. We can modify the second term, $\Delta G_{\text {el }}$ by taking into account the classical model of non-Gaussian effect. ${ }^{16}$ At higher $\alpha_{\|}$, it can be expressed as ${ }^{17}$

$$
\begin{aligned}
\frac{\Delta G_{\mathrm{el}}}{k_{B} T}= & C_{1} \frac{N_{c}}{2}\left(\alpha_{\|}^{2}+2 \alpha_{\perp}^{2}-3\right)+C_{2} \frac{N_{c}}{2}\left(\alpha_{\perp}^{4}+2 \alpha_{\perp}^{2} \alpha_{\|}^{2}-3\right) \\
& +C_{3} \frac{N_{c}}{2}\left(\alpha_{\|}^{4}+2 \alpha_{\perp}^{4}-3\right)+\cdots .
\end{aligned}
$$

Now, we can discuss the swelling curves of NIPA gels by using Eq. (1) in conjunction with the extended Flory-type free energy. ${ }^{3}$ We assume that $C_{2}$ is much smaller than $C_{1}$, and $C_{i}$ 's $(i=3,4,5, \ldots)$ are negligibly small. It should be noted that the latter assumption can lead the semiempirical model by the Mooney-Rivlin equation. ${ }^{18,19}$ The free energy of gels under uniaxial elongation can be described as

$$
\begin{aligned}
\frac{\Delta G}{k_{B} T}= & N_{s}[\ln (1-\phi)+\chi \phi]+\frac{N_{c}}{2}\left[\ln \left(\frac{\phi}{\phi_{0}}\right)\right. \\
& \left.-3+C_{1}\left(\alpha_{\|}^{2}+2 \alpha_{\perp}^{2}-3\right)+C_{2}\left(\alpha_{\perp}^{4}+2 \alpha_{\perp}^{2} \alpha_{\|}^{2}-3\right)\right] \\
& +N_{c} f \ln \phi-\frac{F l_{0}}{k_{B} T}\left(\alpha_{\|}-1\right)
\end{aligned}
$$

where $N_{s}$ is the number of solvent molecules, $N_{C}$ is the number of polymer chains, $\phi\left(\phi_{0}\right)$ is the volume fraction of polymer (the initial value as prepared), $\chi$ is the polymer- solvent interaction parameter, $f$ is the ionized groups per chain, $F$ is the applied force, and $k_{B}$ is the Boltzmann constant. Analysis of the data was performed in a first approximation by using the following relations ${ }^{3}$

$$
\begin{aligned}
& \frac{\phi_{0}}{\phi}=\frac{V}{V_{0}}=\alpha_{\|} \alpha_{\perp}^{2}, \quad N_{s}=\frac{(1-\phi) V}{\nu_{s}}, \quad \chi=\chi_{1}+\chi_{2} \phi, \\
& \chi_{1}=\frac{\Delta H-T \Delta S}{k_{B} T},
\end{aligned}
$$

where $\nu_{S}$ is the volume occupied by one solvent molecule, $\Delta H$ and $\Delta S$ are the enthalpy and entropy of polymerpolymer contact, and $\chi_{2}$ is a constant. The equilibrium condition, expressed by

$$
\left(\frac{\partial \Delta G}{\partial V}\right)_{\alpha_{\|}}=-\frac{\phi^{2}}{V_{0} \phi_{0}}\left(\frac{\partial \Delta G}{\partial \phi}\right)_{\alpha_{\|}}=0,
$$

determines the relation between $T$ and $\phi$;

$$
\begin{aligned}
T^{-1}= & \frac{\Delta S}{\Delta H}+\frac{k_{B}}{\Delta H}\left[-\frac{\ln (1-\phi)}{\phi^{2}}-\frac{1}{\phi}+\chi_{2}(1-2 \phi)\right] \\
& +\frac{k_{B}}{\Delta H} \frac{\nu_{s} N_{c}}{V_{0} \phi_{0}}\left[\left(\frac{1}{2}+f\right) \frac{1}{\phi}-\frac{C_{1}}{\alpha_{\|}} \frac{\phi_{0}}{\phi^{2}}\right. \\
& \left.\times\left\{1+\frac{C_{2}}{C_{1}}\left(\alpha_{\|}^{2}+\frac{1}{\alpha_{\|}} \frac{\phi_{0}}{\phi}\right)\right\}\right] .
\end{aligned}
$$

First of all, the calculations on the results of the basic swelling curve without elongation were carried out for various values of the parameters by using the numerical method, ${ }^{3,7,9}$ in order to determine the materials constants in Eq. (2), hereafter, $\alpha_{\|}$was changed by using the same constants. The swelling curves for the neutral NIPA gels $(f=0)$ were calculated for several values of $\alpha_{\|}$as shown in Fig. 5(a), which explains the preliminary result of Fig. 2(a). The swelling curves obtained under the larger constrained length above $\alpha_{\|} \sim 4$ were quite successfully described within the phenomenological model on the basis of the extended Flory-type free energy.

Next we will consider the force change with temperature. The restoring force can be expressed by using the above classical model of non-Gaussian effect;

$$
\begin{aligned}
F= & \frac{1}{l_{0}} \frac{\partial \Delta G}{\partial \Delta \alpha_{\|}}=k_{B} T \frac{N_{c}}{l_{0}}\left[C_{1}\left(\alpha_{\|}-\frac{\phi_{0}}{\phi} \frac{1}{\alpha_{\|}^{2}}\right)\right. \\
& \left.+C_{2}\left\{\frac{\phi_{0}}{\phi}-\left(\frac{\phi_{0}}{\phi}\right)^{2} \frac{1}{\alpha_{\|}^{3}}\right\}\right] .
\end{aligned}
$$

By combining Eqs. (4) and (5), we have the relation between $T$ and $F$, which is shown in Fig. 5(b). This figure qualitatively describes the experimental results shown in Fig. 2(b): $\alpha_{\|}^{*}$ is in between $\alpha_{\|}^{*}=3$ and 4 , and $F$ discontinuously decreases at the transition point for $\alpha_{\|}>\alpha_{\|}^{*}$. However, the jump at the transition point does not increase rapidly above $\alpha_{\|}^{*}$, in other words, the absolute value of $F$ in the swollen state does not increase at the larger $\alpha_{\|}$. The discrepancy with the experimental observations might be due to the underes- 


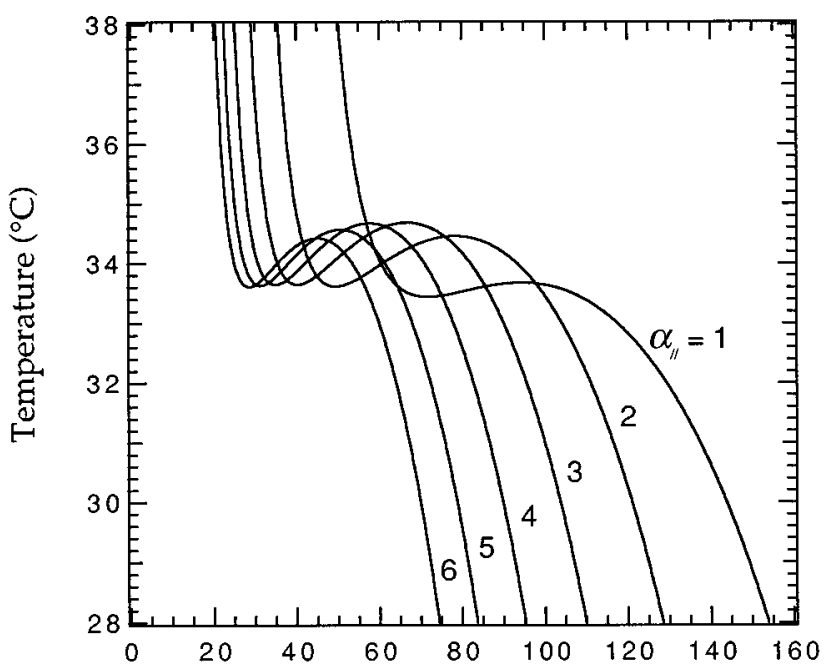

(a)

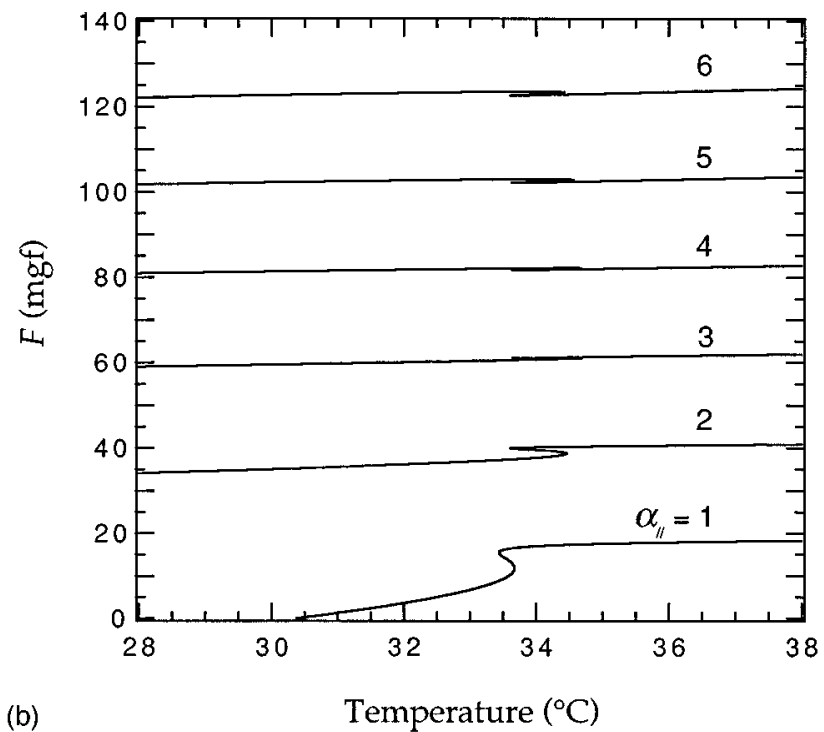

FIG. 5. Theoretical equilibrium diameter (a) and force (b) vs temperature of the neutral NIPA gel for various $\alpha_{\|}$are constructed using the equations in the text. The results are qualitatively consistent with the experimental observations.

timation of the effective number of $N_{C}$. One can say that the expression of Eq. (5) is only a qualitative approximation but an essential equation which indicates that the elastic term of the non-Gaussian chains have an important role to explain the transition behavior.

The theoretical consideration in this section is based on the simple Flory elasticity. On the other hand, the recent theoretical arguments ${ }^{22-24}$ have taken the fact into consideration that the thermally activated nucleation is strongly suppressed in the bulk gels, ${ }^{22}$ therefore the volume phase transition takes place at the stability limit (spinodal) of the swollen state of the gel. It is possible to explain the discrepancy between the experiments and the mean-field theory in terms of the newly developed thermodynamic analysis. ${ }^{22-24}$ In order to test the theory, not only the mechanical but also the scattering experiments are desirable in the same gel. This is a subject for future investigations.

\section{Characters of the strongly stretched non-Gaussian chains}

The effects of the uniaxial stretch on the swelling behavior remind us of the swelling curve of the ionized gels without mechanical constraint. ${ }^{15}$ The uniaxial elongation, though the compression is along the diameter direction, has a similar role as the ionization to induce the positive osmotic pressure in the NIPA gels. The present results indicate that the deformation by stretching can add an internal pressure in the gel, which brought the gel state deeper into the unstable region enhancing the discontinuous transition. At the transition point, the gel can discontinuously shrink through the hydrophobic interaction, which produces the steplike increase in the force. This consideration can explain the increment of the transition temperature as well as the discontinuous increase of the force at the transition point in the small $\alpha_{\|}$, but not for the large $\alpha_{\|}$where the elastic response becomes non-Gaussian $^{5,13}$ and some complement effects should have an important role.

Evidence for the existence of the crossover elongation ratio $\alpha_{\|}^{*}$ can be attributed to the strong uniaxial deformation where chains should be stretched from their equilibrium endto-end distance to something approaching their full length. In the case of the weak stretching at a swollen state, the elongation of the chain decreases the disorientational entropy of the chains and thus, in turn, increases the free energy which produces a restoring force ${ }^{20,21}$ the entropy of the chains decreases, while that of the water molecules increases, and the total entropy increases at the transition point. On the other hand, under the strong stretching, the decrement of the entropy will saturate to the full extended value, and the entropy does not decrease anymore, which can explain the saturation of the transition temperature. However, this is not enough to explain the observations above $\alpha_{\|}^{*}$ : a slight decrease of the transition temperature and the negative change in the force at the transition point. It is important to take into consideration at least two unique elastic properties in this hydrophobic system at large deformation. One is the volume change by stretching and the other is the deviation of the cross-link positions from affine deformation.

The gel volume can be determined by the osmotic pressure due to the excluded volume, which depends on the polymer concentration. Therefore if the uniaxial stress is applied, the volume could be changed. As is shown in Fig. 2(b), the volume of the swollen phase increases with $\alpha_{\|}$, while the increment of the collapsed phase is very small. This is because the repulsive interaction between polymer networks of the swollen state is much stronger than that of the collapsed state, therefore the balance between the interactive and repulsive forces in the swollen state could be changed by the strong external force. In other words, the network is hydrophilic in the swollen state, which is easily affected by the deformation, while in the collapsed state it is hydrophobic, which is stable under the deformation. Moreover, we can 
consider the deviation from affine deformation in terms of the molecular interaction. When $\alpha_{\|}$is approaching to $\alpha_{\|}^{*}$, the deformation become highly anisotropic, which results in bringing the chains closer together and thus enhancing the transverse intrachain interaction. The strong stretching reduces the probability of the longitudinal interchain interaction. In particular, the transverse hydrophobic interaction on neighboring chains can be enhanced at the transition point. These properties could explain the origin of the complement effects observed dominantly above $\alpha_{\|}^{*}$, and it is desirable to have microscopic information from another technique such as scattering experiments under the large deformation.

Finally, it should be noted that the absolute value of the transition temperature as well as the swelling ratio are very reproducible in the same gel. The respective value is the same within the present experimental accuracy even in going from the largest deformation back to the linear range. Such reversibility does not occur when the saturation is due to strain-induced plastic deformation. We believe that the large elongation in the present study might change permanently the chain structure in the molecular level. However, it does not change the network structure in the intermediate size level, such as microdomain structure, which could determine the macroscopic elastic properties of polymer gels.

\section{CONCLUSION}

We could strongly stretch the cylindrical $N$-isopropylacrylamide gels of submillimeter diameter along the uniaxial direction at $30{ }^{\circ} \mathrm{C}$ (a swollen state), the ratio of the stretched length to the equilibrium length at rest, $\alpha_{\|}$is $1 \leqslant \alpha_{\|} \leqslant 6$. Under various $\alpha_{\|}$we have measured both the diameter and the force to keep the longitudinal length constant as functions of temperature. The transition temperature as well as the change in the force at the transition point for $4 \leqslant \alpha_{\|} \leqslant 6$ are quite different from those for $1 \leqslant \alpha_{\|} \leqslant 3.5$. It was suggested that there is a crossover deformation ratio $\alpha_{\|}^{*}$ between the smaller and the larger deformations. Below $\alpha_{\|}^{*}$ the decrease in the disorientational entropy has an important role, while above $\alpha_{\|}^{*}$ the intermolecular interactions due to the non-
Gaussian chains are essential. These observations were qualitatively described by the phenomenological model taken into account the non-Gaussian effect. In spite of the difference between the model network structure and the actual one, the swelling properties have been described by the mean-field theory based on the simple Flory elasticity. The present observation represents the effect of limited extensibility of chains on the elastic properties of hydrophobic polymer gels, therefore it is obviously of great importance with respect to the evaluation of non-Gaussian theories of the elasticity of polymer gels.

\section{ACKNOWLEDGMENTS}

This work has been supported by a Grant of SCF from Science and Technology Agency, Japan, and by Ministry of Education, Science and Culture in Japan.

${ }^{1}$ K. Dusek and D. Patterson, J. Polym. Sci. 6, 1209 (1968)

${ }^{2}$ T. Tanaka, Phys. Rev. Lett. 40, 820 (1978)

${ }^{3}$ S. Hirotsu and A. Onuki, J. Phys. Soc. Jpn. 58, 1508 (1989).

${ }^{4}$ S. Hirotsu, J. Chem. Phys. 94, 3949 (1991).

${ }^{5}$ P. Pincus, Macromolecules 9, 386 (1976).

${ }^{6}$ P.-G. de Gennes, Scaling Concepts in Polymer Physics (Cornell University Press, Ithaca, 1979).

${ }^{7}$ A. Suzuki and S. Kojima, J. Chem. Phys. 101, 10003 (1994).

${ }^{8}$ A. Suzuki and T. Tanaka, Nature (London) 346, 345 (1990).

${ }^{9}$ A. Suzuki, Adv. Polym. Sci. 110, 199 (1993).

${ }^{10}$ H. M. James and E. Guth, J. Chem. Phys. 11, 455 (1943).

${ }^{11}$ S. Daoudi, J. Phys. (Paris) 38, 1301 (1977).

${ }^{12}$ S. Alexander and Y. Rabin, J. Phys. 2, SA313 (1990).

${ }^{13}$ P. Pekarski, A. Tkachenko, and Y. Rabin, Macromolecules 27, 7192 (1994).

${ }^{14}$ Y. Hirokawa and T. Tanaka, J. Chem. Phys. 81, 6379 (1984).

${ }^{15}$ S. Hirotsu, Y. Hirokawa, and T. Tanaka, J. Chem. Phys. 87, 1392 (1987).

${ }^{16}$ K. Dusek and W. Prins, Adv. Polym. Sci. 6, 1 (1969).

${ }^{17}$ K. J. Smith, Jr., A. Ciferri, and J. J. Hermans, J. Polym. Sci. A 2, 1025 (1964).

${ }^{18}$ M. Mooney, J. Appl. Phys. 19, 433 (1948)

${ }^{19}$ R. S. Rivlin, Philos. Trans. R. Soc. London, Ser. A 241, 379 (1948).

${ }^{20}$ L. R. G. Treloar, The Physics of Rubber Elasticity, 2nd ed. (Clarendon, Oxford, 1958)

${ }^{21}$ S. Candau, J. Bastide, and M. Delsanti, Adv. Polym. Sci. 44, 27 (1982).

${ }^{22}$ A. Onuki, Phys. Rev. A 38, 2192 (1988).

${ }^{23}$ K. Sekimoto, Phys. Rev. Lett. 70, 4154 (1993)

${ }^{24}$ S. Panyukov and Y. Rabin, Macromolecules 29, 8530 (1996). 